\title{
A mini outburst from the nightside of comet 67P/Churyumov-Gerasimenko observed by the OSIRIS camera on Rosetta
}

\author{
J. Knollenberg ${ }^{1}$, Z. Y. Lin $^{16}$, S. F. Hviid ${ }^{1}$, N. Oklay ${ }^{4}$, J.-B. Vincent ${ }^{4}$, D. Bodewits ${ }^{7}$, S. Mottola ${ }^{1}$, M. Pajola ${ }^{6}$,
} H. Sierks ${ }^{4}$, C. Barbieri ${ }^{9}$, P. Lamy ${ }^{5}$, R. Rodrigo ${ }^{21,22}$, D. Koschny ${ }^{17}$, H. Rickman ${ }^{3,20}$, M. F. A'Hearn ${ }^{7}$, M. A. Barucci ${ }^{10}$, J. L. Bertaux ${ }^{11}$, I. Bertini ${ }^{6}$, G. Cremonese ${ }^{9}$, B. Davidsson ${ }^{3}$, V. Da Deppo ${ }^{12}$, S. Debei ${ }^{8}$, M. De Cecco ${ }^{13}$, S. Fornasier ${ }^{10}$, M. Fulle ${ }^{14}$, O. Groussin ${ }^{5}$, P. J. Gutiérrez ${ }^{15}$, W.-H. Ip ${ }^{16}$, L. Jorda ${ }^{5}$, H. U. Keller ${ }^{1,2}$, E. Kührt ${ }^{1}$, J. R. Kramm ${ }^{4}$, M. Küppers ${ }^{18}$, L. M. Lara ${ }^{15}$, M. Lazzarin ${ }^{9}$, J. J. Lopez Moreno ${ }^{15}$, F. Marzari ${ }^{9}$, G. Naletto ${ }^{19,6,12}$, N. Thomas ${ }^{23}$, C. Güttler ${ }^{4}$, F. Preusker ${ }^{1}$, F. Scholten ${ }^{1}$, and C. Tubiana ${ }^{4}$

${ }^{1}$ Institute of Planetary Research, DLR, Rutherfordstrasse 2, 12489 Berlin, Germany e-mail: joerg.knollenberg@dlr.de

2 Institute for Geophysics and Extraterrestrial Physics, TU Braunschweig, 38106 Braunschweig, Germany

3 Department of Physics and Astronomy, Uppsala University, 75120 Uppsala, Sweden

${ }^{4}$ Max-Planck-Institut für Sonnensystemforschung, Justus-von-Liebig-Weg, 337077 Göttingen, Germany

5 Aix Marseille Université, CNRS, LAM (Laboratoire d'Astro-physique de Marseille) UMR 7326, 13388 Marseille, France

Center of Studies and Activities for Space (CISAS) "G. Colombo", University of Padova, via Venezia 15, 35131 Padova, Italy

Department for Astronomy, University of Maryland, College Park, MD 20742-2421, USA

Department of Mechanical Engineering - University of Padova, via Venezia 1, 35131 Padova, Italy

9 University of Padova, Department of Physics and Astronomy, vicolo dell'Osservatorio 3, 35122 Padova, Italy

${ }^{10}$ LESIA, Obs. de Paris, CNRS, Univ Paris 06, Univ. Paris-Diderot, 5 place J. Janssen, 92195 Meudon, France

11 LATMOS, CNRS/UVSQ/IPSL, 11 boulevard d'Alembert, 78280 Guyancourt, France

12 CNR-IFN UOS Padova LUXOR, via Trasea 7, 35131 Padova, Italy

13 UNITN, Università di Trento, via Mesiano, 77, 38100 Trento, Italy

14 INAF-Osservatorio Astronomico di Trieste, via Tiepolo 11, 34143 Trieste, Italy

15 Instituto de Astrofísica de Andalucía - CSIC, 18080 Granada, Spain

16 Institute for Space Science, National Central University, 32054 Chung-Li, Taiwan

17 Research and Scientific Support Department, European Space Agency, 2201 Noordwijk, The Netherlands

18 ESA/ESAC, PO Box 78, 28691 Villanueva de la Cañada, Spain

19 Department of Information Engineering - University of Padova, via Gradenigo 6, 35131 Padova, Italy

20 PAS Space Research Center, Bartycka 18A, 00716 Warszawa, Poland

21 International Space Science Institute, Hallerstraße 6, 3012 Bern, Switzerland

22 Centro de Astrobiología, CSIC-INTA, 28850 Torrejón de Ardoz, Madrid, Spain

23 Physikalisches Institut, University of Bern, Sidlerstrasse 5, 3012 Bern, Switzerland

Received 13 November 2015 / Accepted 13 July 2016

\section{ABSTRACT}

Context. On 12 March 2015 the OSIRIS WAC camera onboard the ESA Rosetta spacecraft orbiting comet 67P/ChuryumovGerasimenko observed a small outburst originating from the Imhotep region at the foot of the big lobe of the comet. These measurements are unique since it was the first time that the initial phase of a transient outburst event could be directly observed.

Aims. We investigate the evolution of the dust jet in order to derive clues about the outburst source mechanism and the ejected dust particles, in particular the dust mass, dust-to-gas ratio and the particle size distribution.

Methods. Analysis of the images and of the observation geometry using comet shape models in combination with gasdynamic modeling of the transient dust jet were the main tools used in this study. Synthetic images were computed for comparison with the observations.

Results. Analysis of the geometry revealed that the source region was not illuminated until $1.5 \mathrm{~h}$ after the event implying true nightside activity was observed. The outburst lasted for less than one hour and the average dust production rate during the initial four minutes was of the order of $1 \mathrm{~kg} / \mathrm{s}$. During this time the outburst dust production rate was approximately constant, no sign for an initial explosion could be detected. For dust grains between $0.01-1 \mathrm{~mm}$ a power law size distribution characterized by an index of about 2.6 provides the best fit to the observed radiance profiles. The dust-to-gas ratio of the outburst jet is in the range 0.6-1.8.

Key words. comets: general - comets: individual: 67P/Churyumov-Gerasimenko - methods: numerical 


\section{Introduction}

The OSIRIS camera onboard ESA Rosetta spacecraft (S/C) observes the nucleus of comet $67 \mathrm{P} /$ Churyumov-Gerasimenko (67P) and the surrounding near nucleus coma continuously since the close approach of Rosetta in May 2014 (Sierks et al. 2015). OSIRIS is a two-camera system comprising of a narrow angle camera (NAC) for high resolution imaging of the nucleus and a wide angle camera (WAC) with a field-of-view of $11.3^{\circ} \times 12.1^{\circ}$ optimized for coma observations (Keller et al. 2007). Both cameras are equipped with a total of 26 filters that allow imaging the comet in different colors between $250 \mathrm{~nm}$ and $1000 \mathrm{~nm}$.

Cometary outbursts are characterized by a rapid increase in brightness usually followed by a gradual decay over the next hours or days. They are a common phenomenon in comets (Hughes 1999) but the driving mechanism is still poorly understood. They occur on very different scales, from very large events like the giant outburst of comet 17P/Holmes in 2007 (Lin et al. 2009) which are easily accessible by ground based observations down to the mini-outbursts which may only be detected by observing spacecraft. The Deep Impact spacecraft detected ten such mini-outbursts on its approach to 9P/Tempel-1 during a time span of only two months (Farnham et al. 2007), a surprisingly large number of such events, especially if one takes into account that the monitoring was not continuous. Tubiana et al. (2015a) reported a mini outburst of 67P which occurred around 30 April 2014 that was observed during the Rosetta approach phase. The OSIRIS observations discussed in the following are for the first time providing information on the initial phase of a mini-outburst and, furthermore, prove unambiguously that outbursts can occur also during night.

\section{Observations}

The observations discussed below were acquired by the OSIRIS WAC on 12 March 2015 at a heliocentric distance of 2.12 AU. The S/C distance to the comet at that time was $80 \mathrm{~km}$ and the phase angle of the observations $50^{\circ}$. A typical OSIRIS gas observation sequence was run where images in several gas filters and the associated continuum filters are taken in the shortest possible amount of time in order to support the co-registration of images. A list of the images acquired during this sequence and used for this study is given in Table 1 . All images listed in Table 1 were acquired in $4 \times 4$ binning mode which results in a pixel scale of $32 \mathrm{~m}$ at the nucleus.

Figure 1 shows the development of the dust jet in four continuum images, three taken in the UV-375 nm and the last one in the VIS-610 nm continuum filter. The nucleus part of the images has been strongly overexposed in favor of a better signalto-noise $(\mathrm{S} / \mathrm{N})$ ratio in the surrounding coma. The jet shows up for the first time in the image taken at 7:13:08 UTC. It then grows rapidly in brightness during the next few minutes. Besides the rather broad main jet expanding under a position angle of $146^{\circ}$ with respect to the Sun (Sun is up, position angle is counted counter-clockwise from the Sun direction) a secondary highly collimated dust filament is also visible. The next image sequence was taken about $1 \mathrm{~h}$ later and showed that the outburst had ceased, although a weak diffuse dust signal above the estimated source region could still represent a remnant of the outburst dust cloud. OSIRIS observations were continued on the next day and we acquired more images at very similar conditions with respect to rotational phase and illumination conditions about $25.5 \mathrm{~h}$ (or two comet rotational periods, note that no OSIRIS images were acquired at the next rotational period following the outburst) after the nightside jet was detected. In this case the rotational phase at the start of the corresponding imaging sequence was slightly shifted by only $9 \mathrm{~min}$ compared to the previous sequence but no clear sign of activity from the nightside could be observed. Furthermore, during the following month the OSIRIS observation plan provided further opportunities (on 23-25 March, and 12/13 Apr 2015) for repeated observations of the source region under comparable illumination conditions, but in neither of these cases activity from Imhotep was detected (for the definition of the regions on 67P see Thomas et al. 2015). These observational facts show that the activity was not related to the diurnal cycle of the comet.

\section{Image processing}

All images were pre-processed using the OSIRIS calibration pipeline (Tubiana et al. 2015b), which includes bias subtraction, flat fielding, conversion from digital units to radiance, and distortion correction. For the filters used in this analysis the uncertainty of the measured radiance is less than $1.7 \%$. To prepare the quantitative analysis of the evolving dust distribution a few additional processing steps were performed. During the five minutes of observation the change in the observing geometry is rather small but not fully negligible for the present purpose. The $\mathrm{S} / \mathrm{N}$ ratio is only sufficient to detect the jet in the inner part of the coma. Therefore, in a first step a $128 \times 128$ subframe was extracted from each original image, magnified by a factor of four, and manually cleaned from bright spots due to stars, cosmic rays, or big dust grains. Thereafter, a co-registration of the images was performed by determining the optimum linear shift between image pairs and aligning the images accordingly. Then, in order to eliminate the coma background as much as possible, difference images were computed between the cleaned images taken after the jet started and a reference image acquired in the same filter 1-2 min before the outburst. The resulting three difference images which form the basis of all subsequent analysis are shown in Fig. 2.

\section{Results}

\subsection{Illumination conditions}

Looking at the images of the developing jet one immediately gets the impression that this jet emanates from a non-illuminated part of the surface. In order to prove this hypothesis we computed the detailed observation geometry at the time of image acquisition by using the best shape model of the comet currently available (a merged shape model combined of the SPG based SHAP4S (Preusker et al. 2015) and the SPC based SHAP5 (Jorda et al. 2016) models) together with the relevant SPICE kernels supplied by ESA providing the trajectory and S/C attitude. Using these tools the Sun incidence angle for the whole shape model was computed and displayed in Fig. 3. The projection shown on the left of Fig. 3 is close to the image plane and the Sun is located in the direction of the yellow arrow, upwards and approximately $40^{\circ}$ out of the image plane corresponding to a phase angle of $50^{\circ}$. The chosen color table shows increasing incidence angles from red to blue colors, and black corresponds to incidence angles $>90^{\circ}$, that is to non-illuminated regions. It is evident that the whole "foot" part of the nucleus (Imhotep and surrounding regions) was in shadow during the relevant period of time, thus the dust jet emanates certainly from the non-illuminated part of the nucleus. Moreover, by considering the sense of rotation of the comet it is evident that the apparent source region has not been 
Table 1. OSIRIS WAC images used in this study.

\begin{tabular}{lllll}
\hline \hline Image filename & Time [UTC] & Filter & $t_{\exp }[\mathrm{s}]$ & Remarks \\
\hline WAC_2015-03-12T07.11.20.095Z_ID30_1397549001_F13 & $07: 11: 20$ & UV-375 & 45 & reference image, no jet \\
WAC_2015-03-12T07.12.10.348Z_ID30_1397549003_F18 & $07: 12: 10$ & VIS-610 & 0.45 & reference image, no jet \\
WAC_2015-03-12T07.12.13.500Z_ID30_1397549004_F14 & $07: 12: 13$ & CN & 52.5 & still no jet \\
WAC_2015-03-12T07.13.08.057Z_ID30_1397549005_F13 & $07: 13: 08$ & UV-375 & 45 & jet detection \\
WAC_2015-03-12T07.15.18.870Z_ID30_1397549007_F13 & $07: 15: 18$ & UV-375 & 45 & factor 7.7 increase in brightness \\
WAC_2015-03-12T07.16.09.296Z_ID30_1397549009_F18 & $07: 16: 09$ & VIS-610 & 0.45 & \\
\hline
\end{tabular}

Notes. The times indicated are the start times of the exposures.

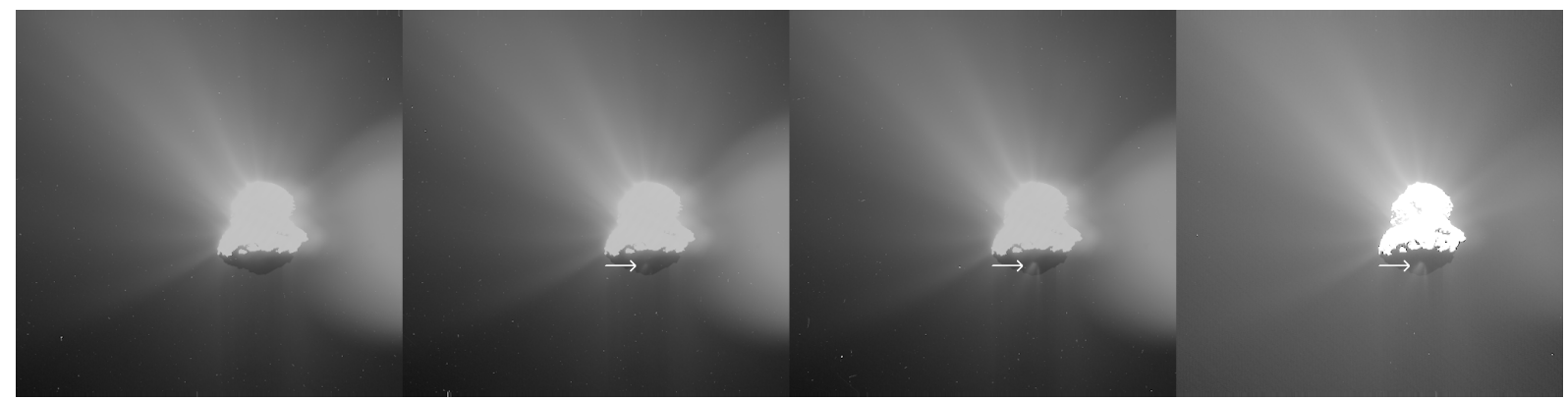

Fig. 1. Development of a dust jet emanating from the Imhotep region (indicated by the arrow) on the night side of the comet. The left image was taken in the UV-375 nm filter on 12 March 2015 at 7:11:20 UTC before the outburst, the second image from the left shows the first appearance of the new jet at 7:13:08 (UV-375 nm), and images 3 and 4 were acquired at 7:15:08 and 7:16:09 in the UV-375 nm and VIS-610 nm filters, respectively. A logarithmic scaling was applied to enhance the visibility of the faint structures. The projected Sun direction is upwards and the FOV is $16.4 \times 16.4 \mathrm{~km}$. The broad bright feature in the right part of the images is an artefact due to the primary ghost of the WAC.

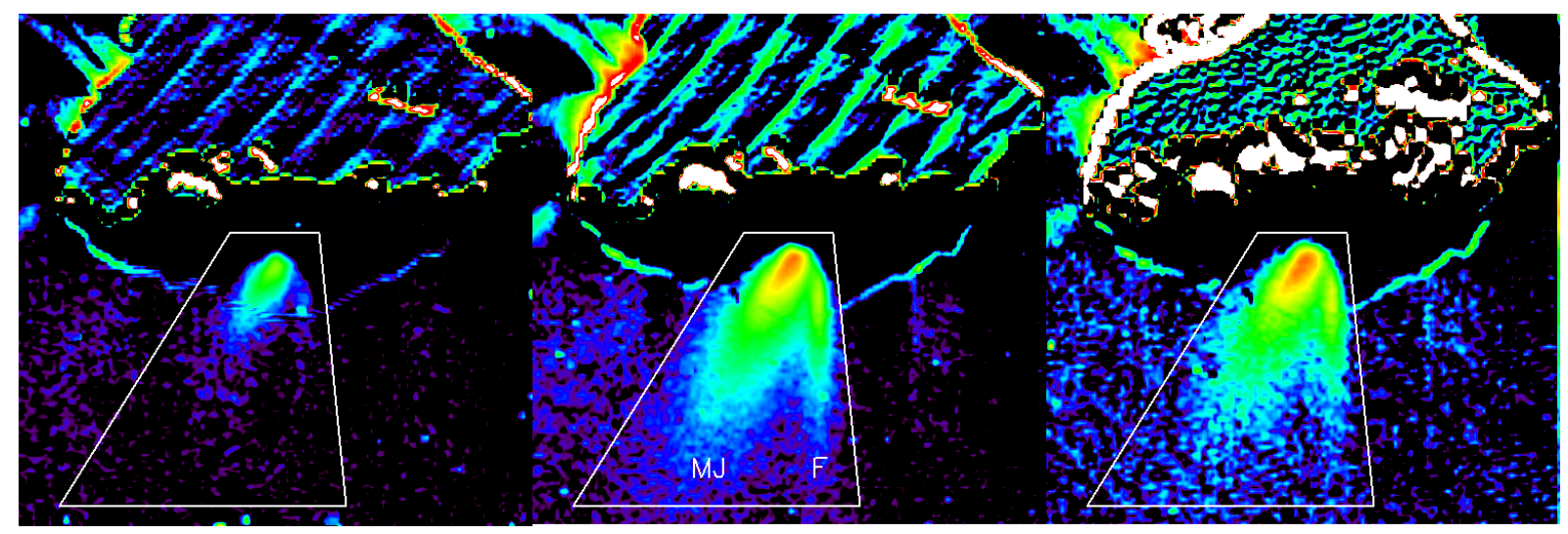

Fig. 2. Co-registered, cleaned and magnified (factor 4) difference images. The image taken before the outburst (left panel in Fig. 1) has been subtracted from the three continuum images acquired after the outburst started (panels 2-4 in Fig. 1). The polygonal area is defined as the jet region used to evaluate the brightness evolution of the outburst. MJ indicates the main jet, $F$ stands for filament.

illuminated since several hours, hence it can be concluded that this jet is an example of real nightside activity.

With images only taken from one viewing direction where the jet can be unambiguously identified a stereoscopic reconstruction of the source region on the comet is obviously not possible. Nevertheless, some geometrical and gas dynamic arguments can be made to provide an estimate where the source could be located. Due to the specific geometry at the time of observation it is highly probable that the source lays somewhere in the shadow above the area where the dust jet is seen in the image. That the broad main jet and the collimated dust filament appear at the same time is a strong indication that both features are connected and originate from the same source region. Moreover, assuming that the outflow is nearly radial from the origin the source point can be determined geometrically by intersection of the two axes of the main jet and the filament, respectively.
It should be understood that the triangulation provides a virtual source point only, meaning that all dust outflow seems to come from this point, which does not necessarily need to be located on the nucleus surface.

\subsection{Determination of the source region}

To determine the direction (position angles) of the jet and filament axes and their respective uncertainties the following method was applied. Starting with an initial guess for the source location the angular positions of the brightness maxima of jet and filament were determined as a function of distance from the source. Then a linear least squares fit was used to estimate the position angles of jet and filament and new axes directions were derived. An updated source location was then determined by computing the intersection point of the two axes. 


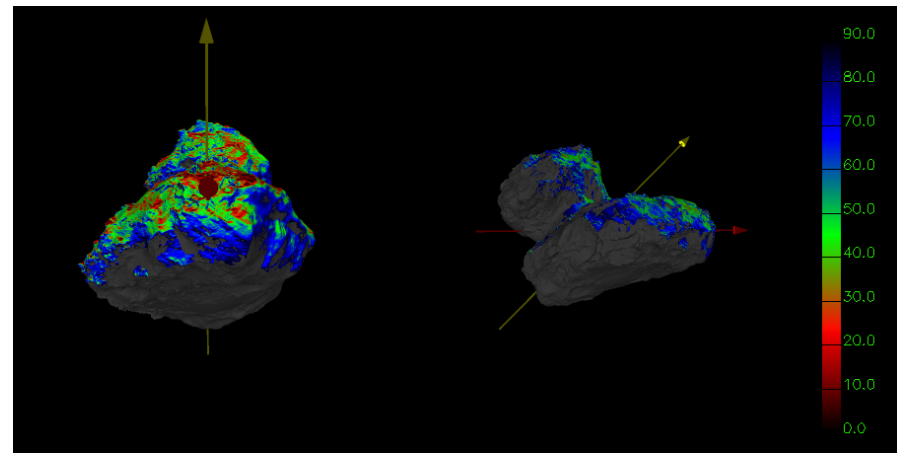

Fig. 3. Sun incidence angle (red: $0^{\circ}$, blue: $90^{\circ}$ ) at the time of outburst on 12 March 2015 07:13:08. Left side: projection approximately corresponding to the image geometry, Sun upwards. The whole foot of the big lobe of the nucleus is in shadow (incidence angle $>90^{\circ}$ ) including the possible jet source regions. Right side: side view, red arrow indicates direction to the observer, yellow arrow direction to the Sun.

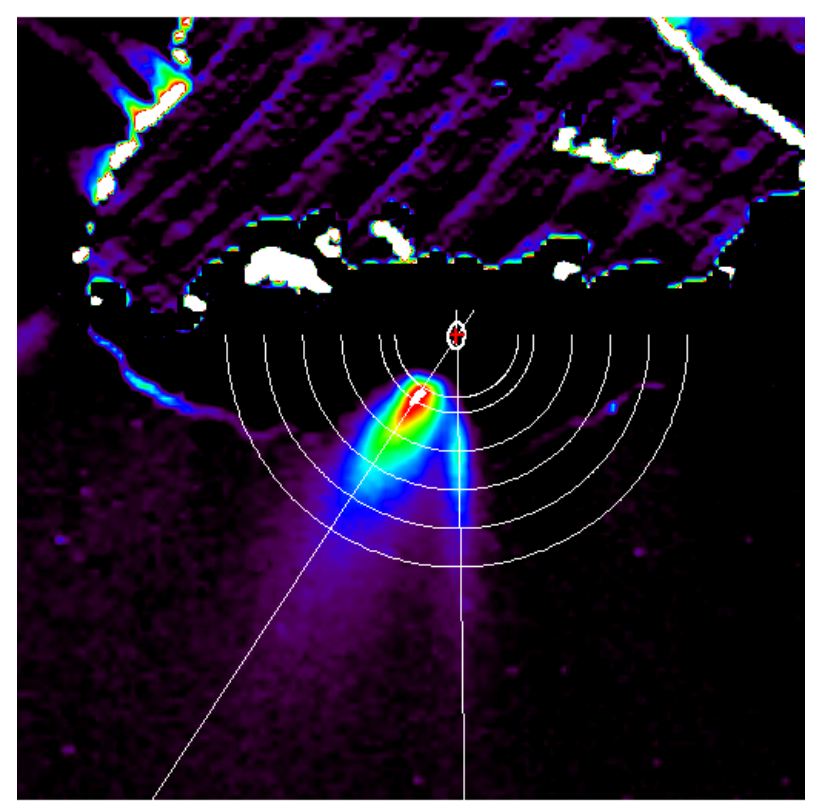

Fig. 4. Difference image between the exposures taken at 7:11:20 (before the outburst) and 7:16:09 (after the outburst) in the UV-375 nm filter clearly showing the broad main jet and a secondary narrow filament. The red cross shows the suspected source point constructed under the assumption that the two jets originate from the same location and the ellipse gives the $2 \sigma$ uncertainty. The half circles indicate the distances used for the angular radiance distributions plotted in Fig. 5.

This procedure converges after 2-3 iterations for a given range of distances from the source. Numerical tests revealed that the choice of the distance range used for fitting has an influence on the results due to some systematic deviations between data and model. For example, a slight systematic curvature of the main jet for distances larger than about $1000 \mathrm{~m}$ was found which is probably caused by the rotation of the nucleus. Therefore, a Monte carlo approach was used to estimate the uncertainty of the triangulation. The minimum and maximum distances used for fitting were sampled from a random uniform distribution between $300-400 \mathrm{~m}$ and $800-1000 \mathrm{~m}$, respectively. The resulting values for the position angle are $146.5^{\circ}+/-1.2^{\circ}$ for the main jet and $181.0^{\circ}+/-1.0^{\circ}$ for the filament where the given uncertainties correspond to $1 \sigma$. These numbers translate into an uncertainty of the triangulation of $10 \mathrm{~m}$ in $x$-direction and $30 \mathrm{~m}$ in $y$-direction in the

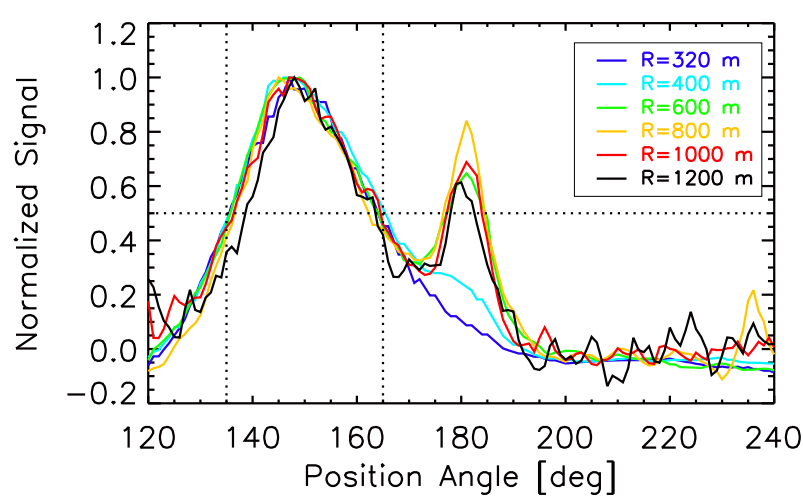

Fig. 5. Normalized angular radiance distribution as a function of position angle on circles around the estimated source point. The angular profile resembles a Gaussian and for distances between $300 \mathrm{~m}$ and $1000 \mathrm{~m}$ from the source the FWHM of the jet is approximately constant at about $30^{\circ}$.

image plane. Further uncertainties of the projected source location are due to the finite extension of the source, the unknown depth of the virtual source point below the surface, and the imperfect correction of the motion (mainly due to rotation) of the nucleus between images. Note that due to the observation geometry (the average surface normal of Imhotep and the line-of-sight form an angle of about $75^{\circ}$ ) and the associated projection effects the source diameter gives basically an uncertainty in $x$-direction whereas the source depth contributes mostly to the uncertainty in $y$-direction. Since typical dimensions of small jet sources and the depth of the virtual sourcepoint of a dust jet below the surface are both of the order of a few tens of meters the combined effect of these uncertainties was modeled by a uniform probability distribution with $\sigma=25 \mathrm{~m} / \sqrt{3}$ in both directions. The factor $\sqrt{3}$ stems from the computation of the standard deviation of a uniform distribution in accordance with the GUM (2013). The uncertainty caused by the imperfect co-registration of the images due to the nucleus movement within the considered time frame was estimated to $\sigma=10 \mathrm{~m} / \sqrt{3}$. All uncertainties mentioned above were then added quadratically to provide the combined uncertainties in the $x$ - and $y$-directions in the image plane. The best fit of the jet axes, the resulting location of the source in the image, and the $2 \sigma$ uncertainty ellipse are shown in Fig. 4.

The plausibility of the above solution for the location of the source can further be checked by the following gas dynamic consideration. Since the source of the jet is located on the not illuminated part of the nucleus it can be assumed that the jet expands either into a vacuum or into a very low pressure background gas. The brightness distribution of the main jet is nearly symmetric with respect to the direction of maximum brightness, thus resembling closely what would be expected from an axisymmetric flow projected onto the image plane. It is known in gas dynamics (Koppenwallner et al. 1986) that the stationary solution of the expansion of a free axisymmetric supersonic gas jet into a vacuum can well be approximated in the far field by a so called virtual source flow, where all streamlines are directed radially away from a common source point. This description applies in the far field, at distances larger than $\sim 10$ times the source diameter and here the density distribution as a function of the angle with the jet axis can well be described by a Gaussian. Though this is strictly true only for gas jets, this rule is also a good approximation for the dust component of a dusty gas jet as shown by Knollenberg (1994). Therefore, in a steady state, one would expect that the angular width of the jet 


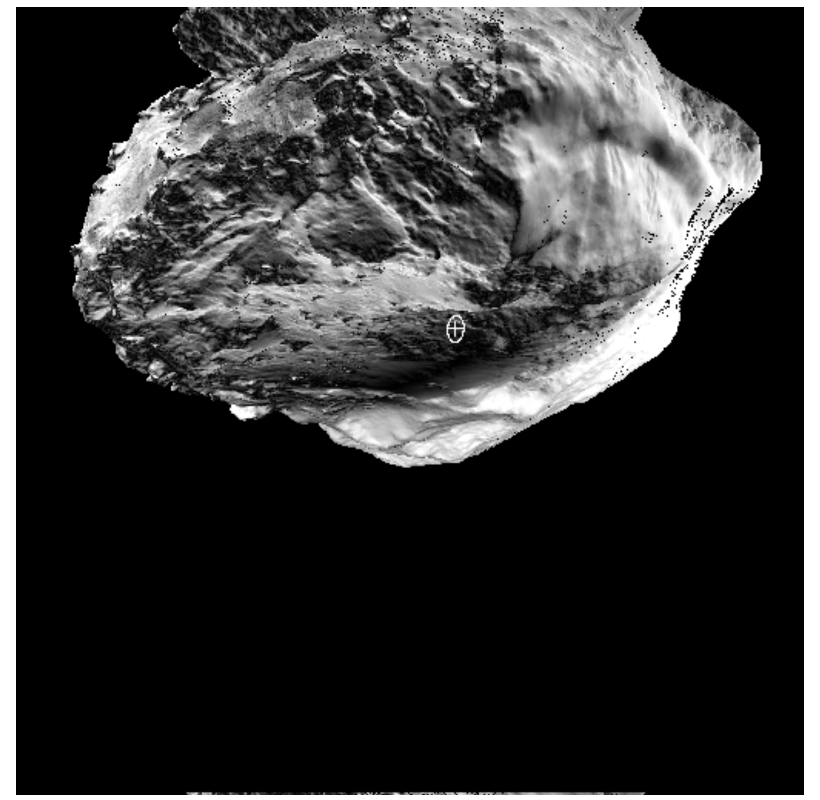

Fig. 6. Synthetic image of $67 \mathrm{P}$ where the location of the estimated source region is marked by the white cross. The $2 \sigma$ uncertainty is indicated by the ellipse. Note that an artificial light source was used to show the otherwise not illuminated region.

is independent of the distance from the virtual source point. That this is indeed the case can be seen in Fig. 5 where the normalized angular brightness distributions at different distances from the source (as indicated by the half circles in Fig. 4) are plotted. Clearly, the shape of the azimuthal profile resembles a Gaussian. Furthermore, between $300 \mathrm{~m}$ and $1000 \mathrm{~m}$ distance from the estimated source the full-width-half-maximum (FWHM) of the jet is about $30^{\circ}$ and nearly constant, for larger distances the width of the jet decreases slightly. The latter fact can be explained by the transient nature of the phenomenon, the jet has not yet established steady state at larger distances.

The probable source region on the nucleus can be made visible in a synthetic image (Fig. 6) which was computed for the observational geometry at the time when the nightside activity occurred, but with an additional artificial light source that illuminates also the shadowed regions. One sees that the source is located inside a depression with rougher terrain close to the smooth plain of the Imhotep region.

\subsection{Properties of the source region}

The determined location of the source area at a latitude of $12.1^{\circ}$ and a longitude of $168.5^{\circ}$ in the Cheops reference frame (Preusker et al. 2015) and its regional context on the nucleus is given in Fig. 7. Here, a simulated WAC image provides the global overview and NAC images acquired during a color mapping sequence from 5 September 2014 (months before the outburst; acquired at an altitude of $43 \mathrm{~km}$ with a pixel scale of $0.8 \mathrm{~m}$ ) provide high resolution information of the source region. The NAC image presented in the middle panel of Fig. 7 is an RGB-like image where three individual NAC images taken in the filters IR, Hydra and Green are coded to the RGB channels of the image, respectively. The bottom panel of Fig. 7 displays the zoom-in of our source region. Roundish features are surrounded by brighter green material, while the rest of the surface is in gray-brown color. The red cross indicates the best estimate of

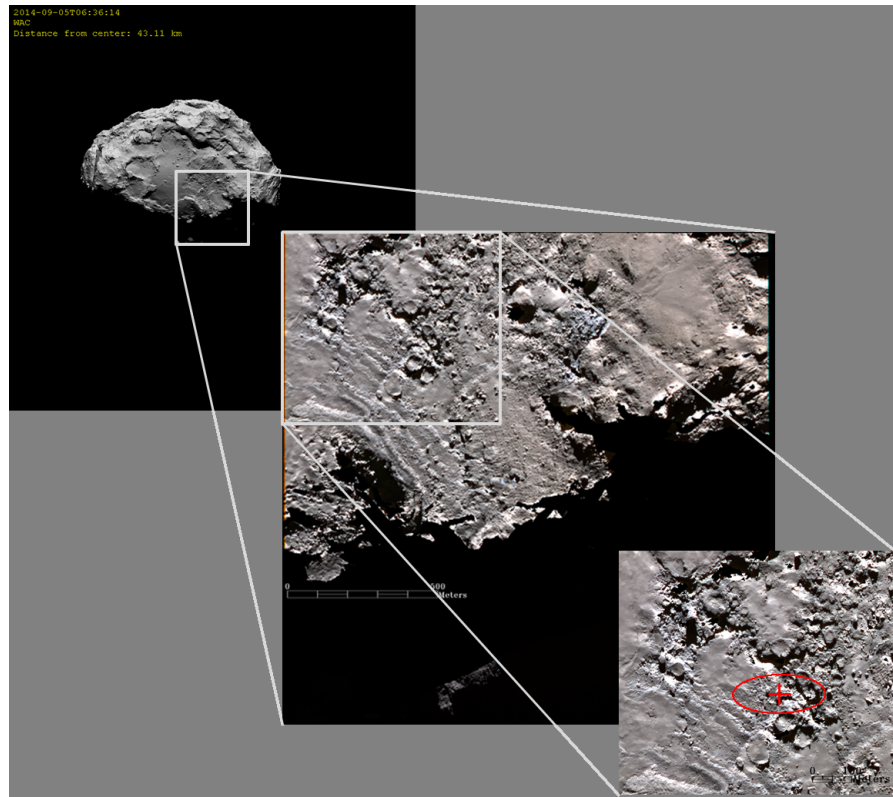

Fig. 7. Simulated WAC view of the image taken on 5 September 2014 around 06:36:14 UTC (months before the outburst) with the NAC FOV marked in gray square (top panel), NAC image in RGB (middle panel) with the source region in RGB (bottom panel). R: IR, G: Hydra, B: Green. The best estimate of the jet source point is marked by the red cross and the ellipse provides the $2 \sigma$ uncertainty of the source location.

the jet source point and the ellipse provides the $2 \sigma$ uncertainty of the source location.

The source region consists mainly of rocky terrain (Auger et al. 2015) but some smooth parts are also visible. The constructed virtual source point of the jet itself lies inside a fractured part of rocky terrain in the close vicinity of roundish features of different sizes. The roundish features are ranging in diameter from 2-60 $\mathrm{m}$ and were interpreted by Auger et al. (2015) as ancient degassing conduits.

Oklay et al. (2016) investigated large parts of the Imhotep region using spectral techniques and the potentially active regions are detected based on spectral slopes. The detected source region of the outburst (bottom panel of Fig. 7) was one of the potential activity sources detected by Oklay et al. in the images taken on 5 September 2014, long before the outburst was observed. In this study, we extract the source Fig. 7) and investigate the spectral properties of the region separately. Details of the spectral analysis methods and the definitions can be found in Oklay et al. (2016).

The source region detected in this study is displayed as a decorrelation stretched image in Fig. 8a) which emphasizes the variety in colors in the region. Variegation is clear in the vicinity of the roundish features once compared with the rest of the visible surface. Spectral slopes are presented in Fig. 8b) showing that these regions belong to the active regions group defined by Oklay et al. (2016), due to their lower spectral slopes $(10-12 \% / 100 \mathrm{~nm}$ between $536 \mathrm{~nm}$ and $882 \mathrm{~nm})$ than their vicinity $(12-15 \% / 100 \mathrm{~nm})$, which is in the group of mixed regions. The latter correspond to either surfaces partially covered by material ejected from active spots or inactive areas peppered with small bright spots, which are currently interpreted as minor ice deposits. The best estimate of the source point is located in the active region class although the uncertainty in the determination of the source cannot exclude that the jet comes from the mixed regions. 

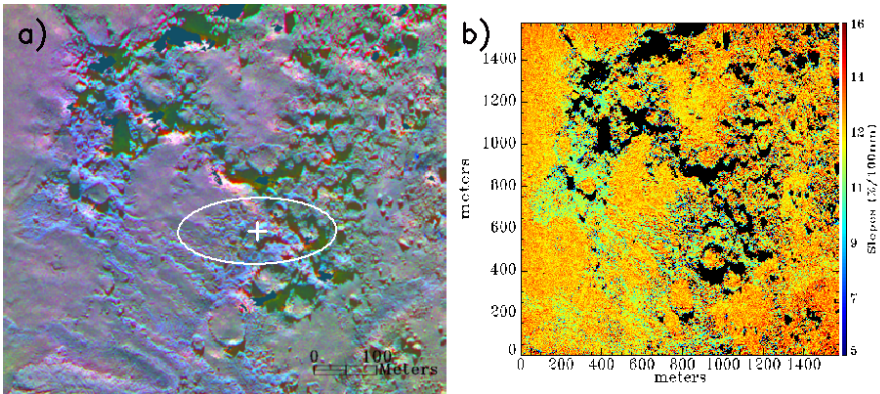

Fig. 8. Detected outburst region, a) decorrelation stretched image, b) spectral slope, the white cross indicates the best estimate of the jet source point and the ellipse provides the $2 \sigma$ uncertainty.

\subsection{Dust cross section and mass}

The specific radiance received from the optically thin dust coma can be expressed as:

$L_{\lambda}=f_{\text {coma }} \frac{p_{\lambda}}{\pi} \frac{\phi(\alpha)}{\phi_{0}} \frac{f_{\theta, \lambda}}{r_{\mathrm{h}}^{2}}$

where $p_{\lambda}$ is the geometric albedo of the dust particles at wavelength $\lambda, \phi(\alpha)$ is the phase function at phase angle $\alpha, f(\theta, \lambda)$ is the specific solar flux (in $\mathrm{W} \mathrm{m} \mathrm{mm}^{-2}$ at $1 \mathrm{AU}$ ) and $r_{\mathrm{h}}$ is the heliocentric distance in $\mathrm{AU}$, and $f_{\text {coma }}$ is the dust filling factor, that is the fraction of a pixel covered by dust. The assumption of neglectable optical thickness is justified by the observation that even the maximum coma brightness at the base of the most prominent jets is only about $3 \%$ of the average nucleus brightness.

By integrating the radiance over an image area containing all dust from the jet (polygonal area in Fig. 2) the total cross section of the dust jet can be determined. Assuming that the geometric albedo of the dust particles is similar to that of the nucleus we use $p_{375 \mathrm{~nm}}=0.039$ (Fornasier et al. 2015), the ratio of the flux at $50^{\circ}$ phase to the backscattered flux is $\phi(\alpha) / \phi(0)=0.4$ (Kolokolova et al. 2004), $f_{375}=1.06 \mathrm{~W} \mathrm{~m}^{-2} \mathrm{~nm}^{-1}$, and $r_{\mathrm{h}}=$ 2.12 AU one finds a dust cross section of $35 \mathrm{~m}^{2}$ in the first (difference) image showing the new jet, which then increased by a factor of $7.7\left(269 \mathrm{~m}^{2}\right)$ in the next image acquired about $130 \mathrm{~s}$ later.

In order to convert the cross section to a dust mass assumptions about the size distribution of the dust grains are required. A close flyby of Rosetta at 67P was performed in March 2015. The evaluation of the combined data acquired be GIADA and OSIRIS on single dust grains during this flyby (Fulle et al. 2016) confirmed qualitatively the result already found at larger heliocentric distances by Rotundi et al. (2015), the mass distribution seems to be flat for sizes larger than $\sim 500 \mu \mathrm{m}$ (corresponding to a power law differential size distribution $n\left(r_{\mathrm{d}}\right) \sim r_{\mathrm{d}}^{-g}$ with an index of $g=4$ ) whereas the size distribution index is shallower than 3 for the smaller grains. If the jet particles follow the same law (here we assumed $g=2.5$ for the small particles, a minimum particle radius of $1 \mu \mathrm{m}$, and a maximum particle radius of $3 \mathrm{~cm}$ ) and using a constant bulk density of $1000 \mathrm{~kg} \mathrm{~m}^{-3}$ for all particles a total dust mass of about $300 \mathrm{~kg}$ was produced during the first three minutes after the outburst became visible. The mass estimate is little dependent on the chosen minimum size, by varying the minimum radius between $100 \mathrm{~nm}$ and $10 \mu \mathrm{m}$ the total dust mass in the outburst varies only between $293 \mathrm{~kg}$ and $321 \mathrm{~kg}$. The influence of the upper cut-off size is somewhat larger, by increasing the maximum radius from $1 \mathrm{~cm}$ to $10 \mathrm{~cm}$ the dust mass increases from 233 to $374 \mathrm{~kg}$. It should also be noted that the true

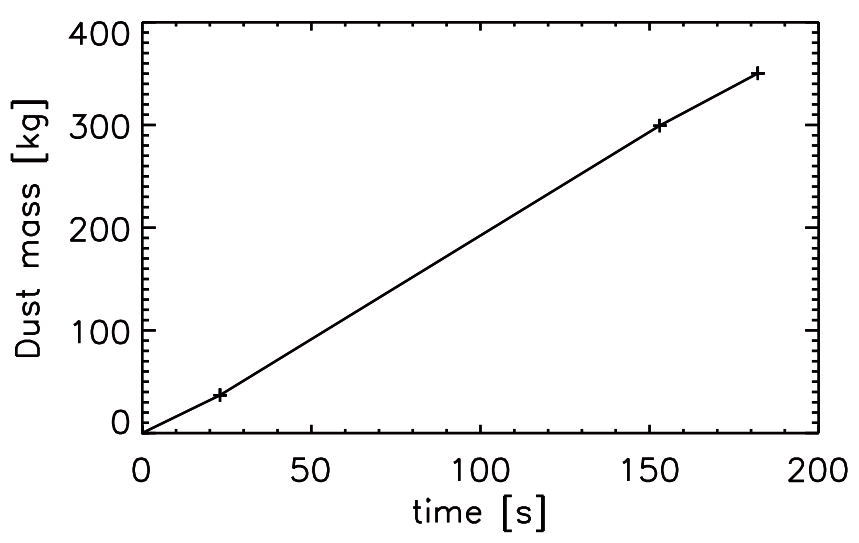

Fig. 9. Dust mass in jet as a function of time assuming a size distribution according to Rotundi et al. (2015).

mass might be larger than the estimates given above because a fraction of the dust might still be hidden in the shadow and some fast moving dust might have alreay left the field-of-view. The dust production of the outburst in absolute terms is rather small, it corresponds to about $1-3 \%$ of the total dust production of the comet at this heliocentric distance (Fulle et al. 2016).

The evolution of the total jet mass over time is depicted in Fig. 9. Here, $t=0$ is the start of the exposure of the first image where the outburst was visible, the time of the data points are taken as the midpoint of the exposures. A linear increase of dust mass as a function of time provides a good approximation to the data which could be interpreted as a continuous constant activity of the source during this time interval. A caveat has to be made here because the 3rd data point was acquired with the VIS-610 nm filter instead of UV-375 $\mathrm{nm}$. To make this observation comparable with the others an assumption about the optical properties of the dust is required. Here, we assumed that the geometric albedo of the nucleus is also representative for the dust at $610 \mathrm{~nm}\left(p_{610 \mathrm{~nm}}=0.057\right)$ and that the backscatter peak is the same as for UV-375 $\mathrm{nm}$, too.

The development of the radial brightness profiles on the axis of the main jet is shown in Fig. 10 which can be used to derive clues about the expansion velocity of the dust. The main source of uncertainty in this case is the remaining offset due to the imperfect background coma subtraction. This offset was quantified by evaluation of the remaining background in regions outside of the jet but located in the lower part of the difference images. The background uncertainty was determined to $2.1 \times 10^{-8} \mathrm{~W} \mathrm{~m}^{-2} \mathrm{~nm}^{-1} \mathrm{sr}^{-1}$ and this value is indicated by the horizontal dotted line in Fig. 10. The two vertical dotted lines show how far the dust has travelled (at least) for the two first difference images acquired in the UV-375 nm filter giving values of $d=1.0 \mathrm{~km}$ for the first and $d=2.1 \mathrm{~km}$ for the second image. The distance of $1.1 \mathrm{~km}$ between the two subsequent exposures was travelled by the dust within $130 \mathrm{~s}$ giving a minimum projected dust speed of $8.5 \mathrm{~m} \mathrm{~s}^{-1}$. Of course, this estimate does not exclude the presence of a certain amount of faster particles which could be hidden in the noise. That there are indeed also faster particles in the jet becomes clear when looking more closely at the brightness profile in the first exposure. Although the exact start of the outburst and the precise location of the source are not known we know that the dust has travelled at least the distance between the point where it becomes visible in the image and $d=1.0 \mathrm{~km}$ during the $45 \mathrm{~s}$ long exposure (note that the $\mathrm{CN}$ image taken immediately before does not show any signs of the outburst). This 


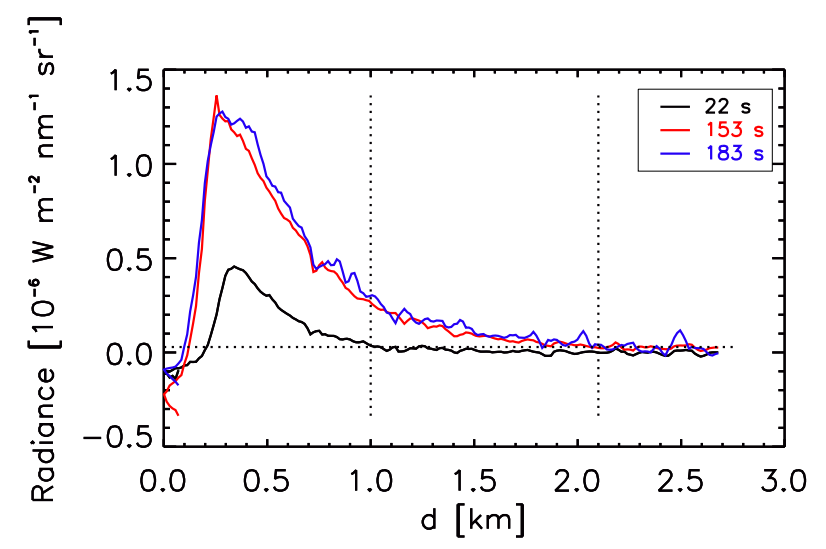

Fig. 10. Radial radiance profiles on the axis of the main jet. The horizontal line indicates the uncertainty level in the background subtraction and the vertical dotted lines indicate the minimum travel distance of dust for the first two exposures. For the 3rd image the much higher noise level is too high for useful analysis. Note that for the 3rd image (blue) acquired in VIS-610nm filter the brightness has been multiplied by $p_{375} * f_{375} / p_{610} * f_{610}$ to make the data comparable in terms of the dust cross section.

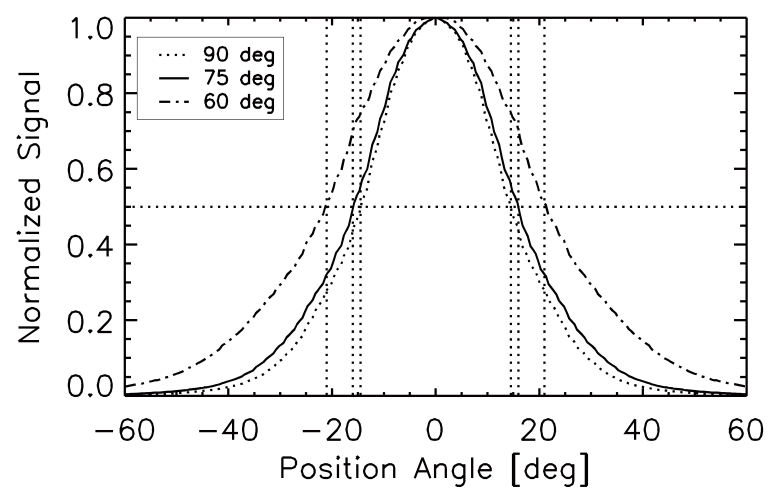

Fig. 11. Normalized radiance of a simulated dust jet as a function of position angle and viewing geometry at a distance of $800 \mathrm{~m}$ from the origin. FWHM increases with decreasing viewing angle (measured between jet axis and observer) and takes values of $29^{\circ}, 32^{\circ}, 42^{\circ}$ for viewing angles of $90^{\circ}, 75^{\circ}$, and $60^{\circ}$, respectively.

consideration results in a velocity of some fraction of the dust $v_{\mathrm{d}}>16 \mathrm{~m} \mathrm{~s}^{-1}$.

\subsection{Dust outflow modeling}

A coupled gas/dust continuum model (Knollenberg 1994) has been applied to further support the interpretation of the above described observations. The time dependent Euler equations for coupled gas and dust flow have been solved using a second order Godunov-type method (Harten et al. 1983) on a fixed grid in axisymmetric polar coordinates (Kitamura 1986). A homogenously active region of $1^{\circ}$ angular width has been assumed on the spherical model nucleus of $2 \mathrm{~km}$ radius and a reservoir outflow boundary condition (Knollenberg 1994) has been specified here assuming an initial Mach number of $M_{0}=1$. Free supersonic outflow has been assumed on the outer boundary set at a cometocentric distance of $20 \mathrm{~km}$. The dust particles are assumed to be initially at rest and the standard equation of motion for a spherical particle in a free molecular flow has been used to model the gas-dust interaction (Kitamura 1986). After the computation of the dust and gas flow field integration along a line of sight was executed to calculate dust column densities for a more meaningful comparison with the images. Because the activity was observed on the nightside we assumed that a gas more volatile than $\mathrm{H}_{2} \mathrm{O}$ is the driver, and, therefore, we chose $\mathrm{CO}_{2}$ described by a molecular mass of 44 AMU and an adiabatic exponent of $5 / 3$ for the gas species. For the simulation of the dust up to 30 sizes with radii ranging between $10 \mu \mathrm{m}$ and $1 \mathrm{~cm}$ with a constant bulk density of $1000 \mathrm{~kg} \mathrm{~m}^{-3}$ were used. Because for the interesting particle size distribution the mass loading effect on the gas flow can be neglected, the results of a single model run could be rescaled for different size distributions.

The use of a continuum model for the gas flow in this case is justified by the rather strong production rates and associated small mean free path values for gas collisions which are required to produce the necessary gas from a small source region. In the models discussed below we are using gas production rates of $10^{22}-10^{23} \mathrm{~m}^{-2} \mathrm{~s}^{-1}$ giving mean free path values of the order of $1-10 \mathrm{~cm}$ and a comet source region of approximately $70 \mathrm{~m}$ diameter resulting in initial Knudsen numbers of the order of $K n=10^{-4}-10^{-3}$. These are sufficiently small for a continuum treatment of the flow. Of course, the Knudsen numbers will increase further away from the source and the gas flow will enter a rarefied transition regime, but the strongest acceleration of the dust particles takes place rather close to the surface. For these reasons, no strong influence is expected on the results for the dust outflow by the use of the hydrodynamic approximation.

Figure 11 shows the normalized column density of a dust jet with a power law size distribution and a differential size index of $g=2.6$ and a maximum particle size of $1 \mathrm{~mm}$ emanating into a vacuum as a function of position angle in the image plane and viewing angle (defined as the angle between the jet axis and the observer) at a radial distance of $800 \mathrm{~m}$ from the source. The characteristic shape approximately resembling a Gaussian is clearly seen and the width of the jet is about $30^{\circ}$ when viewed from the side (jet axis in the image plane, viewing angle $=90^{\circ}$ ), but increasing with decreasing viewing angle. The width of the modeled dust jets is compatible with the observations for viewing angles $>70^{\circ}$, meaning that the axis of the jet is close to the image plane. This is also consistent with dust ejection approximately normal to the surface given that the average surface normal vector of the Imhotep region in inclined by about $75^{\circ}$ with respect to the line-of-sight. There also exists a slight dependence of the jet width on the size distribution index (because smaller particles are better coupled to the gas and show stronger lateral expansion) but for the considered grains with $r_{\mathrm{d}}>10 \mu \mathrm{m}$ the effect is minute.

An interesting point which could be investigated by exploiting the time-dependent capabilities of the code is the effect of a stationary source function compared with a time dependent source function on the observable column density as a function of time. Typical results of these model runs are given in Fig. 12. On the left side the simulated brightness distribution of the jet at times corresponding to the UV-375 nm images are shown for the case of a constant production rate of $5 \times 10^{21} \mathrm{~m}^{-2} \mathrm{~s}^{-1}$ or $1.4 \mathrm{~kg} \mathrm{~s}^{-1}$ total gas production rate at the source. To account for the long exposure times the simulation results were also integrated between $t=0-45 \mathrm{~s}$ for the first and $t=130-175 \mathrm{~s}$ for the second image. As above, a power law differential size distribution characterized by an exponent of $g=2.6$ was chosen for this run. This boundary condition let the outer edge of the dust cloud move at an average velocity of about $9 \mathrm{~m} \mathrm{~s}^{-1}$ while the inner part slowly evolves towards 

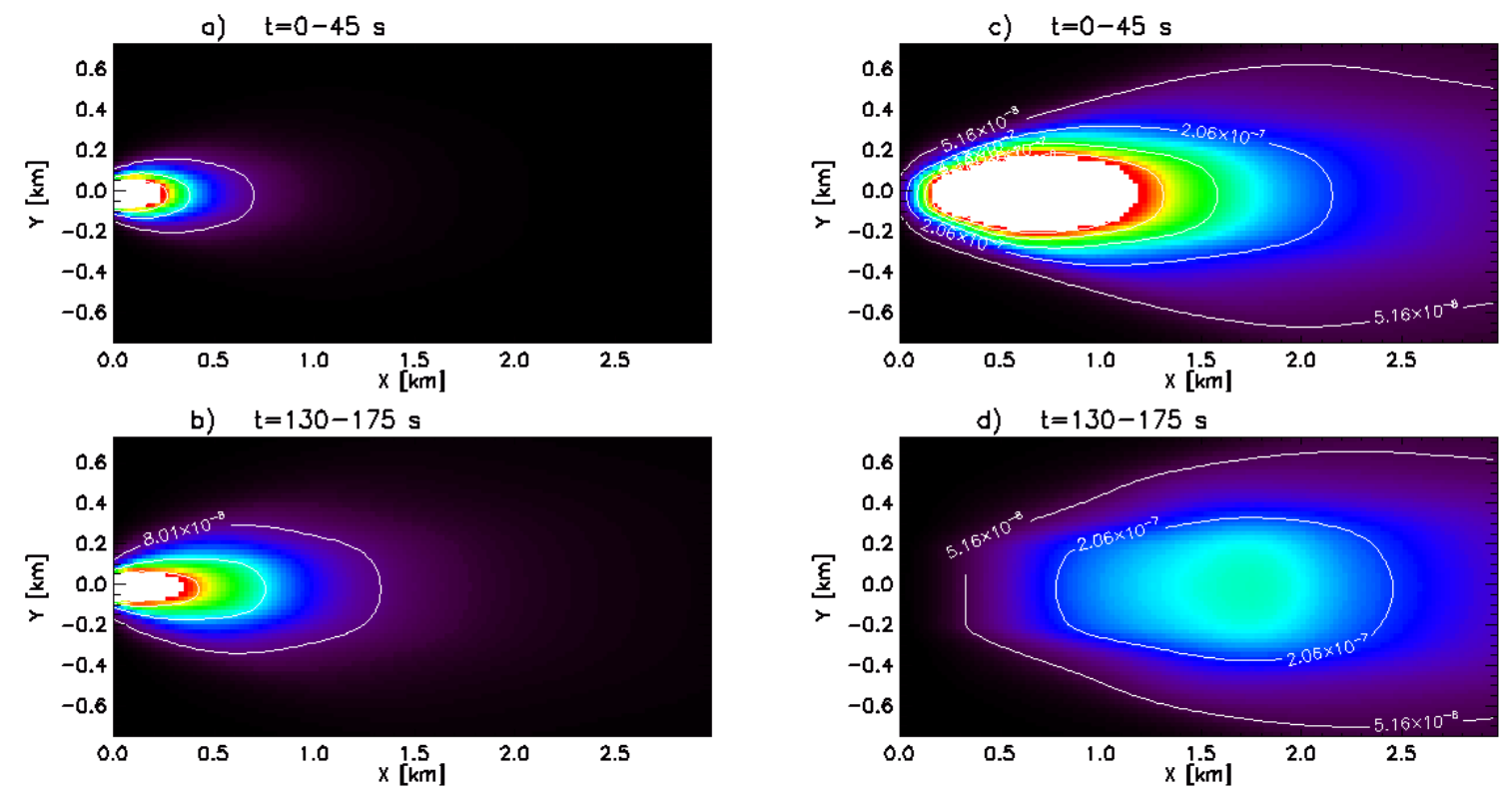

Fig. 12. Simulated images integrated between $t=0-45 \mathrm{~s}$ and $t=130-175 \mathrm{~s}$ (to simulate the long exposures of the UV-375 nm images) for two different source functions. In the left panels $\mathbf{a}$ ) and b) the production rate is kept constant during the whole simulation, on the right (c) and d)) the production was stopped at $t=5 \mathrm{~s}$. In this case the moving dust cloud has detached from the source at $t=130-175 \mathrm{~s}$ and a rather flat brightness distribution can be seen.

a steady state. On the right, we show for comparison the case with a short strong production pulse of $28 \mathrm{~kg} \mathrm{~s}^{-1}$ which is lasting for a duration of about $5 \mathrm{~s}$. In the beginning the simulated image is qualitatively similar except for the higher column density due to the larger production rate but after $150 \mathrm{~s}$ the picture is completely different. The expanding dust cloud has clearly detached from the source and the whole brightness distribution is much flatter than for the constant production case. It should be noted that this general behavior is not dependent on the size distribution, only the distance of the brightness maximum and the degree of flatness changes. Comparing these results with the OSIRIS images it is obvious that the constant production case matches the observations much better.

Since the outflow speed of the dust is a function of particle size, the simulation of the dust cloud can also be used to derive clues about the size distribution. This was achieved by systematically varying the size distribution exponent and the dust production rate and fitting the observed radiance on the axis of the main jet (see Fig. 4 where the jet axis is shown as a white line). Here, we only used the two UV-375 nm difference images for the analysis because these are not dependent on assumptions about the reddening of the dust and have a superior $\mathrm{S} / \mathrm{N}$ ratio compared to the VIS-610 nm image. Furthermore, the last VIS$610 \mathrm{~nm}$ image was taken immediately after the long UV-375 nm expose and thus provides little additional information about the transient development of the jet. Another important point that has to be taken into account is that the dust travels for some time in the shadow cast by the nucleus before it becomes visible in the images (see right side of Fig. 3). The consequence is that the true start time of the event is uncertain. Therefore, the start time was used as another free parameter and also determined simultaneously by the fitting procedure. In the following examples we always used a viewing angle of $75^{\circ}$ which corresponds to a case where the jet leaves its source normally to the average orientation of Imhotep. The distance range chosen for the fit is between $0.4 \mathrm{~km}$ and $2.0 \mathrm{~km}$ from the source, because for shorter distance the dust cloud is partially in shadow whereas for larger distance the measured brightness drops below the uncertainty limit of the observation.

We first tried to match the observations assuming that the size distribution could be described by a single exponent over the whole size range from $10 \mu \mathrm{m}$ to $1 \mathrm{~cm}$, but no good fit was possible with these assumptions. Then we varied the maximum dust radius between $0.5 \mathrm{~mm}$ and $1 \mathrm{~cm}$. Good fits could be achieved with these assumptions for a narrow size range around $a_{\max }=1+/ 0.1 \mathrm{~mm}$ (Fig. 13), a size distribution exponent of $2.6+/-0.2$, a dust production rate of $0.9+/-0.1 \mathrm{~kg} \mathrm{~s}^{-1}$, and a jet start time at $t=-65+/-10 \mathrm{~s}$. This means that the outburst started between 7:11:53 and 7:12:13 UTC and the corresponding dust-to-gas ratio is $D / G=0.66+/-0.07$. With these parameters both radiance profiles of the UV-375 nm difference images acquired at $t=0-45 \mathrm{~s}$ and $t=130-175 \mathrm{~s}$, respectively, are fitted well within the 1-sigma uncertainty of the difference images as shown in Fig. 13.

The fit parameters determined above are not unique, and according to Rotundi et al. (2015) the size distribution of the coma grains of $67 \mathrm{P}$ is better described by a "knee"-like distribution which is characterized by two different exponents, $g<3$ for the small grains and $g \sim 4$ for the larger grains. Therefore, we also tried to fit the observations with such a size distribution. Here, we kept $g=4$ for the large size range constant, but varied the corner grain radius, the exponent of the small grains, and the maximum grain radius. It was found that good fits are indeed possible and that the fit quality is very sensitive to the chosen corner size, only corner sizes close to $0.5 \mathrm{~mm}$ provided fits within the uncertainty range of the observations. Furthermore, the fit quality was not strongly dependent on the maximum dust size. Maximum dust radii between $3 \mathrm{~mm}$ and $1 \mathrm{~cm}$ all match the data equally well. This is due to the low velocity of about $2-4 \mathrm{~m} \mathrm{~s}^{-1}$ of the large particles. This means that most of them have not yet entered the region with a distance $d>0.4 \mathrm{~km}$ where reliable data exists which is not affected by shadowing from the nucleus and, therefore, was used for the analysis. Because of the limited influence of the maximum grain radius on the quality of the fit 


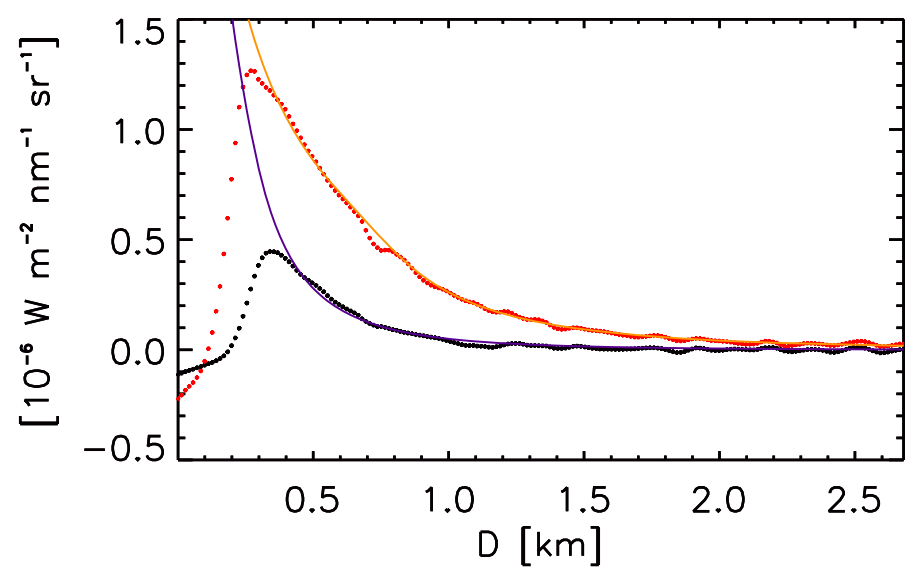

Fig. 13. Model fits of radiance distribution along the jet axis (orange and blue lines) with the data (red and black dots) for the first two (UV-375 nm) difference images. Fit parameters are the size distribution exponent, the dust production rate, and the start time of the outburst. The best fit shown was achieved for an exponent of $g=2.6$ and a start time of $t=-75 \mathrm{~s}$ (zero time defined as start of exposure of first image).

the dust-to-gas ratio is less well constrained and varies between $D / G=1$ for $r_{\max }=2 \mathrm{~mm}$ and $D / G=1.8$ for $r_{\max }=1 \mathrm{~cm}$. The best fit exponent for the grains with radius $<0.5 \mathrm{~mm}$ varies between $g=1.7$ and $g=2.4$ whereas the offset time is again in the range 55-75 s, the same as found for the simple power law size distribution with $r_{\max }=1 \mathrm{~mm}$.

In principle, the viewing angle has also an influence on the derived fit parameters but within the probable range between $70-90^{\circ}$ that is compatible with the observed angular width of the jet the effect of the viewing angle was found to be rather small and within the uncertainties due to the assumptions about the size distribution.

\section{Discussion and conclusion}

The root cause for cometary outbursts is currently not known but several mechanisms are proposed in the literature. Amongst the suggested mechanisms are fluidization of a weakly bound mixture of ice and dust initiated by a phase change boundary between amorphous and crystalline ice (Belton \& Melosh 2009), cryovolcanism (Belton 2008) and impacts of small meteorites (Hughes 1999). They are usually thought to be initiated by a kind of "explosion" (e.g. the conceptual model in Belton 2008) possibly followed by a more or less extended phase of constant or gradually fading activity. In this respect it should be noted that a hypervelocity impact or an explosion on the surface or shallow subsurface produce very similar phenomena (Holsapple 1993), and, therefore, could hardly be distinguished from each other. Both would generate an initial fireball followed by fast ejecta. Thereafter, the considerably longer excavation of a crater produces an ejecta cone which moves from the source location radially outwards, thereby producing ejecta with decreasing speed until the process stops either due to the strength of the material or due to gravity. In our case we have a small event equivalent to a crater diameter of approximately $1 \mathrm{~m}$ (based on the estimate of the produced mass of a few $100 \mathrm{~kg}$ ) which would mean that cratering should happen in the strength dominated regime (even if the strength is only a few $10 \mathrm{~Pa}$ ) and the formation of the crater of approximately $1 \mathrm{~m}$ diameter should be finished in less than $1 \mathrm{~s}$.
Although the onset of the observed small outburst in the Imhotep region of $67 \mathrm{P}$ is indeed rather fast and the observed relatively low speeds of the outflowing dust could be compatible with an explosion in a low strength medium, the appearance of the jet and its evolution in time are not compatible with an explosive origin. The best comparison here can be made with the "Deep Impact" (DI hereafter) on comet 9P/Tempel-1 where a $370 \mathrm{~kg}$ artificial impactor impacted the comet at a speed of $10.2 \mathrm{~km} \mathrm{~s}^{-1}$ causing an impressive ejecta plume (A'Hearn et al. 2005). Although this event was much larger than the one considered here, the impact speed, the target properties, and the cratering regime are applicable.

Although our main jet is quite broad with a FWHM of $\sim 30^{\circ}$, the DI impactor on comet 9P/Tempel-1 generated a much broader plume. This is also typical for cratering in a wide range of materials where ejection angles (measured from the horizontal) between $30-60^{\circ}$ are found (Richardson et al. 2007). This corresponds to full opening angles of typically $90^{\circ}$ which is hardly compatible with our observation. Furthermore, in the modelling section we have shown that the expansion of a dust cloud that is ejected approximately instantaneously and moving with the relevant speeds would result in a considerably flattened brightness distribution in the jet and that the maximum of brightness at the end of the observation sequence should have already detached from the source. This is not compatible with the observations which are instead in accordance with a constant production model. Therefore, we can conclude that the origin of the observed mini outburst was no explosion.

On the contrary, the results of the dust outflow computations presented above, clearly favor a mechanism which is triggered at some point and then produces gas and dust at a more or less constant production rate for some time (at least 4 min and probably less than $30 \mathrm{~min}$ in our case) until the production ceases again. One possibility to create such process involves release of a volatile gas $\left(\mathrm{CO}\right.$ or $\left.\mathrm{CO}_{2}\right)$ trapped in water ice during the passage of a crystallization front changing the phase of the water ice from amorphous to crystalline. According to Prialnik et al. (1993) this can lead to internal pressure build-up which may be large enough to widen the pores, and, dependent on the mechanical material properties of the ice/dust mixture, the final result could be either the opening of large vents with typical diameters of the order of $1 \mathrm{~m}$ which extend from the interior to the surface, or, in extreme cases even lead to catastrophic run-away growth manifesting itself in an explosive outburst. The latter scenario was favored by Belton (2008) to explain mini-outbursts observed in $9 \mathrm{P} /$ Tempel-1 but since we found a non-explosive outburst the former mechanism might apply in our case. Recent work on thermal comet evolution models using lower thermal conductivity values as usually employed in the past find that the crystallization front may be located at rather shallow depths of the order of 0.5 to several meters (Marboeuf \& Schmitt 2014) and also might be locally inhomogeneous dependent on the initial composition and structure of the nucleus (Rosenberg \& Prialnik 2010). These findings make this explanation more attractive in our case because it is difficult to imagine that such a small event has its origin deep inside the nucleus as would be required by older calculations of the depth of the crystallization front.

Another possible mechanism found by Rosenberg \& Prialnik (2010) is that erratic activity and outbursts which occur more or less randomly distributed over the orbit and local time may be the consequence of internal inhomogeneity of the nucleus structure and composition. Although the diverse interactions of their different inhomogeneities are very complex it seems that a low conductivity layer blocking further penetration of the heat wave 
beneath an ice rich upper layer could promote small outbursts. Here, we may have a relation to the areas with low spectral slopes and classified as active by Oklay et al. (2016) which are found in our source region because the low spectral slopes may be indicative of a larger ice content at or close to the surface. It is also remarkable that the suspected source region contains a number of circular features which may be dormant gas conduits and that circular features were also suggested as sources of the outbursts of 9P/Tempel-1 by Belton (2008).

The size distribution exponent found by the dust flow modeling lies in the expected range for $67 \mathrm{P}$ although we cannot definitely be sure about the true size range. This becomes clear by inspection of the equation of motion for the dust particles (Kitamura 1986). The acceleration of a dust particle is proportional to the product of the gas density and the cross section over mass ratio of the particle. This implies that a 10 times smaller particle than used in our simulation would follow exactly the same trajectory if the gas production rate and the corresponding gas density would also be 10 times smaller. Since we have no measurement of the amount of gas ejected we can only derive information about the size dependence but not about the true size. On the other hand, the assumptions made about the albedo variations of the dust between the $375 \mathrm{~nm}$ and the $610 \mathrm{~nm}$ filters are compatible with normally found dust colors in the coma of 67P (Cremonese et al. 2016) which is an indication that the ejected particles are typical for the comet. It is worth noting that the observed brightness distribution cannot be fitted with the assumption of a single characteristic size, the power law size distribution works considerably better. The best estimate for the size distribution exponent is $g=2.6+/-0.2$ if a power law distribution with a maximum grain radius of $1 \mathrm{~mm}$ is assumed, but the observations are also compatible with a size distribution with a "knee" characterized by two different exponents for the smaller and the larger particles, as found by Rotundi et al. (2015) for the coma of $67 \mathrm{P}$ at heliocentric distances $>3.4 \mathrm{AU}$. The dust-to-gas ratio is about 0.7 for $a_{\max }=1 \mathrm{~mm}$ but increases up to $D / G=1.8$ if the jet has a Rotundi-like size distribution with a maximum grain radius of $1 \mathrm{~cm}$. It is worth noting that in contrast to the real grain size (which scales with the unknown gas production of the outburst) the determined $D / G$ ratios are not dependent on the assumptions about the gas production rate. The derived $D / G$ ratio for the outburst is remarkably lower than the value of 2-6 found by Rotundi et al. (2015) for the average coma dust, especially if one considers that a nightside outburst is most probably driven by higher volatile species like $\mathrm{CO}_{2}$ or $\mathrm{CO}$. This points to the existence of localized pockets with an enriched abundance of ices including highly volatile species in the shallow subsurface of Imhotep.
Acknowledgements. OSIRIS was built by a consortium of the Max-PlanckInstitut für Sonnensystemforschung, in Göttingen, Germany, CISAS-University of Padova, Italy, the Laboratoire d'Astrophysique de Marseille, France, the Instituto de Astrofísica de Andalucia, CSIC, Granada, Spain, the Research and Scientific Support Department of the European Space Agency, Noordwijk, The Netherlands, the Instituto Nacional de Técnica Aeroespacial, Madrid, Spain, the Universidad Polite?hnica de Madrid, Spain, the Department of Physics and Astronomy of Uppsala University, Sweden, and the Institut für Datentechnik und Kommunikationsnetze der Technischen Universität Braunschweig, Germany. The support of the national funding agencies of Germany (DLR), France (CNES), Italy (ASI), Spain (MEC), Sweden (SNSB; Grant No. 74/10:2), and the ESA Technical Directorate is gratefully acknowledged. H. Rickman was also supported by Grant No. 2011/01/B/ST9/05442 of the Polish National Science Center. We thank the ESA teams at ESAC, ESOC and ESTEC for their work in support of the Rosetta mission.

\section{References}

Auger, A. T., Groussin, O., Jorda, L., et al. 2015, A\&A, 583, A35, 13 A'Hearn, M. F., Belton, M. J. S., Delamere, W. A., et al. 2005, Science, 310, 258 Belton, M. J. S. 2008, Icarus, 200, 280

Belton, M. J. S., \& Melosh, J. 2009, Icarus, 198, 189

Cremonese, G., Simioni, E., Ragazzoni, R., et al. 2015, A\&A, 588, A59, 8

Farnham, T. L., Wellnitz, D. D., Hampton, D. L., et al. 2007, Icarus, 187, 26

Fornasier, S., Hasselmann, P. H., Barucci, M. A., et al. 2015, A\&A, 583, A30

Fulle, M., Marzari, F., Della Corte, V., et al., 2016, AJ, 821, 1, 19

Harten, A., Lax, P. D., \& van Leer, B. 1983, Soc. Indus. Applied Math. Rev., 25,35

Holsapple, K. A. 1993, Ann. Rev. Earth Planet. Sci., 21, 333

Hughes, D. W. 1999, Possible mechanisms for cometary outbursts, eds. R. L. Newburn Jnr., M. Neugebauer, J. Rahe, Comets in the Post-Halley Era, 2, (Dordrecht: Kluwer Academic Publishers), 825

Jorda, L., Gaskell, R., Capanna, C., et al. 2016, Icarus, 277, 257

JCGM 2013, Evaluation of measurement data - Guide to the expression of uncertainty in measurement and supplements, ed. J. C.f.G.i. Metrology (Sèvres, France: BIPM)

Keller, H. U., Barbieri, C., Lamy, P., et al. 2007, Space Sci. Rev., 128, 433

Kitamura, Y. 1986, Icarus 66, 241

Knollenberg, J. 1994, Ph.D. Thesis, Georg-August Universität Göttingen, Germany

Kolokolova, L., Hanner, M., Levasseur-Regourd, A. C., \& Gustafson, B. A. S. 2004, in Comets II, eds. M. C. Festou, H. U. Keller, H. A. Weaver (University of Arizona Press), 577

Koppenwallner, G., Boettcher, R. D., Detleff, G., \& Legge, H. 1986, ESAESTEC ESA-SP 265

Lin, Z. Y., Lin, C. S., Ip, W.-H., et al. 2009, AJ, 138 2, 625

Marboeuf, U., \& Schmitt, C. 2014, Icarus, 242, 225

Oklay, N., Vincent, J.-B., Fornasier, S., et al. 2016, A\&A, 586, A80

Preusker, F., Scholten, F., Matz, K. D., et al. 2015, A\&A, 583, A33

Prialnik, D., Egozi, U., Bar-Nun, A., Podolak, M., \& Greenzweig, Y. 1993, Icarus, 106, 499

Richardson, J. E., Melosh, H. J., Lisse, C. M., et al. 2007, Icarus, 190, 357

Rotundi, A., Sierks, H., della Corte, V., et al. 2015, Science, 347, Iss. 6220

Rosenberg, E. D., \& Prialnik, D. 2010, Icarus, 209, 753

Sierks, H., Barbieri, C., Lamy, P., et al. 2015, Science, 347, Iss. 6220

Thomas, N., Sierks, H., Barbieri, C., et al. 2015, Science, 347, Iss. 6220

Tubiana, C., Snodgrass, C., Bertini, I., et al. 2015a, A\&A, 573, 11

Tubiana, C., Güttler, C, Kovacs, G., et al. 2015b, A\&A, 573, A62 